\title{
The Productivity and Characteristics of Iranian Biomedical Journal (IBJ): A Scientometric Analysis
}

\author{
Hassan Asadi ${ }^{1}$ and Ehsan Mostafavi ${ }^{2 *}$ \\ ${ }^{1}$ Deputy of Research, Technology and Education, Research Section, Pasteur Institute of Iran, \\ Tehran, Iran; ${ }^{2}$ Department of Epidemiology and Biostatistics, Research Centre for Emerging and \\ Reemerging Infectious Diseases, Pasteur Institute of Iran, Tehran, Iran
}

Received 3 June 2018; revised 7 June 2018; accepted 9 June 2018

\begin{abstract}
Background: Scientometrics is a discipline that analyzes scientific publications to explore the structure and growth of science. In this work, the quantitative evaluation of the productivity of the Iranian Biomedical Journal (IBJ) is reviewed. Methods: The analysis was done based on a cross-sectional descriptive and an analytical scientometric study. Data were collected from PubMed, Scopus, and Scimago Databases (2000-2017). Scopus and Scimago were used for data search and feature analysis. Analyzed scientometric indicators included the number of citations, publications, CiteScore, SJR (Scimago Journal Rank), SNIP (source normalized impact per paper), self-citation, and Q (quartile) trend. Results: The evaluation of 586 documents, published in IBJ from 2000 to 2017, revealed that most of these documents (99.7\%) have been published in the areas of biochemistry, genetics, and molecular biology, which yielded to an upgrade in Journal Q ranking from Q4 (in 2000) to Q2 (in 2016). Conclusion: Nearly all of the scientometric indicators, evaluated in this study, were found on the rise. Therefore, a growing trend from Q2 to Q1 is predicted for the near future. It is recommended that the journal focuses on a specific subject area to improve the indicators and quality of the journal, in a timely manner. DOI: 10.29252/ibj.22.6.362
\end{abstract}

Keywords: Bibliometric analysis, Bibliometrics, Publications

\section{INTRODUCTION}

$\mathrm{S}$ cientific journals play central roles in the scientific community ${ }^{[1]}$. In assessment of scientific performance, bibliometric and citation indicators are among the most important impact measures of scientific literature ${ }^{[2]}$. Routine evaluation of scientific activities of these journals will provide a clear view of journal motion track and the subject areas ${ }^{[3]}$. This evaluation is also important for policymakers and their decision making. Scientometrics is one of the sciences that is used by many researchers in various fields to analyze and measure scientific production using different indicators. Major research issues include the measurement of impact and the reference sets of articles to investigate the impact of journals and institutes, understanding of scientific citations, mapping scientific fields, and the production of indicators for use in policy making and management contexts $^{[4,5]}$.

In this study, a scientometric analysis of Iranian Biomedical Journal (IBJ) was carried out. IBJ is an international bimonthly journal, founded in 1996, and continuously being published since October $1997^{[6]}$. IBJ covers a wide range of disciplines in the fields of biomedicine and laboratory-based experimental biomedical sciences, viz all aspects of biochemistry, genetics, immunology, cell biology, developmental biology, microbiology, molecular biology, pharmacology, and physiology. IBJ is now being indexed by BIOSIS, PubMed, MEDLINE, Scopus, BIOBASE, Embase, Google Scholar, IranMedex, ISC, Magiran, and Scientific Information Database 
(SID) ${ }^{[7,8]}$. IBJ is the publication of Pasteur Institute of Iran (PII), a member of Institute Pasteur International Network, which includes 33 research institutes around the world, and a network of research and expertise to fight against infectious diseases ${ }^{[9]}$.

The objectives of this study were to categorize IBJ documents, based on discipline, year of publication, country territory etc. and to evaluate the pertaining scientometric indices such as CiteScore, SJR (Scimago Journal Rank), SNIP (source normalized impact per paper), citation, self-citation, and total citation.

\section{MATERIALS AND METHODS}

In this paper, attempts have been made to explore IBJ from 2000 to 2017 (an 18-year period), using scientometric indicators, including the number of citations, number of publications, CiteScore, SJR, SNIP, self-citation, Q (quartile trend, distribution of contributions, documents (by authors, year, type, and subject area), and citation. This study covers 586 articles of 33 published issues, during this time period. MS-Excel software was used for data analysis. Both Scopus and Scimago databases were employed for data retrieval and analyses.

CiteScore is defined as the number of citations received by a journal in the last three years divided by the number of documents indexed in Scopus, during those three years. As an example, the calculation of the CiteScore for year 2016 is done based on the following formula:

\section{CiteScore of a journal in $2016=$}

Total number of citation of a journal for 2013-2015

Number of documents published during 2013-2015

CiteScore is a standard yardstick that provides comprehensive, transparent, and current view of a journal's impact and will guide journal directors in their policy making ${ }^{[10]}$. CiteScore allows to monitor the journal's performance throughout the year, lessening the need to wait until mid-year to see how a journal has performed, during the previous year ${ }^{[11]}$.

SNIP is known as an indicator of the citation impact of scientific journals ${ }^{[12]}$ and using a source-normalized approach corrects differences in citation practices between scientific fields ${ }^{[4,13]}$. The scientometric indices (such as citation impact, CiteScore, and SNIP) of a journal can be extracted from the Scopus Database. SJR is a prestige metric based on the idea that 'all citations are not created equal ${ }^{[14]}$. Every scientific document may receive citations from a wide array of journals. To take into account the prestige of the citing journal, in addition to the number of citations, SJR comes to play. The SJR, therefore, is an indication of quality as well as quantity of the visibility of a certain document.

To extract information such as subject area, documents type etc., we have used the "source" of the Scopus Database. Also, by choosing the name of the journal, we chose each of the indicators for this 18-year period. Q rankings are therefore derived from SJR. Based on SJR, journals are ranked and divided into four subject categories. Q1 denotes the top 25\%, Q2 between top $50 \%$ and top $25 \%$, Q3 top $75 \%$ to top $50 \%$, and Q4 bottom $25 \%$ of impact factor distribution).

\section{Year of publication}

Table 1 depicts the number of documents published in IBJ per year. Overall, 586 research articles were published with an average of 33 articles per year. The maximum numbers of documents/year $(n=56)$ occurred in 2017 , as opposed to the minimum $(n=16)$ in 2000 .

\section{Document type}

In the analysis of the type of records during the studied 18-year period, the absolute majority of the published documents $(\mathrm{n}=578)$ consisted of original articles $(\mathrm{n}=562 ; 97.3 \%)$, followed by review articles $(\mathrm{n}=12,2 \%)$, and letter to the editor $(\mathrm{n}=4,0.7 \%)$. The remaining five were in the form of note $(n=3)$, conference paper $(n=1)$, or editorial $(n=1)$, which were excluded from this analysis.

Table 1. Year-wise distribution of papers published in IBJ

\begin{tabular}{ccccc}
\hline Year & $\begin{array}{c}\text { No. of } \\
\text { volume }\end{array}$ & $\begin{array}{c}\text { No. of } \\
\text { issues }\end{array}$ & $\begin{array}{c}\text { No. of } \\
\text { documents }\end{array}$ & \% \\
\hline 2000 & 1 & 4 & 16 & 3 \\
2001 & 1 & 4 & 25 & 4 \\
2002 & 1 & 4 & 24 & 4 \\
2003 & 1 & 4 & 33 & 6 \\
2004 & 1 & 4 & 33 & 6 \\
2005 & 1 & 4 & 32 & 5 \\
2006 & 1 & 4 & 33 & 6 \\
2007 & 1 & 4 & 38 & 6 \\
2008 & 1 & 4 & 35 & 6 \\
2009 & 1 & 4 & 32 & 5 \\
2010 & 1 & 4 & 24 & 4 \\
2011 & 1 & 4 & 24 & 4 \\
2012 & 1 & 4 & 33 & 6 \\
2013 & 1 & 4 & 34 & 6 \\
2014 & 1 & 4 & 36 & 6 \\
2015 & 1 & 4 & 36 & 6 \\
2016 & 1 & 5 & 42 & 7 \\
2017 & 1 & 6 & 56 & 10 \\
Total & 18 & 75 & 586 & 100 \\
\hline
\end{tabular}




\section{Subject areas}

The subject areas of the published articles were categorized in four groups. Documents may have more than one subject area. The most common areas for IBJ documents were biochemistry, genetics, and molecular biology $(\mathrm{n}=581)$, followed by medicine $(\mathrm{n}=489)$, immunology, and microbiology $(\mathrm{n}=94)$, as well as material science $(\mathrm{n}=94)$.

\section{Citation and document trend}

We made a comparison between the number of citations/year of IBJ (for the period of 2009-2017) and two other national journals, namely Avicenna Journal of Medical Biotechnology and Bioimpact, founded in 2009 and 2010, respectively. These two journals were selected based on their subject areas and Q, according to the Scimago Database. All three journals are active in the fields of biochemistry, genetics, and molecular biology and are now at levels Q2 and Q3. The three journals possess similar document trends for the mentioned nine-year period, as observed in Figure 1A. However, IBJ holds a much higher citation trend for this time period (Fig. 1B).
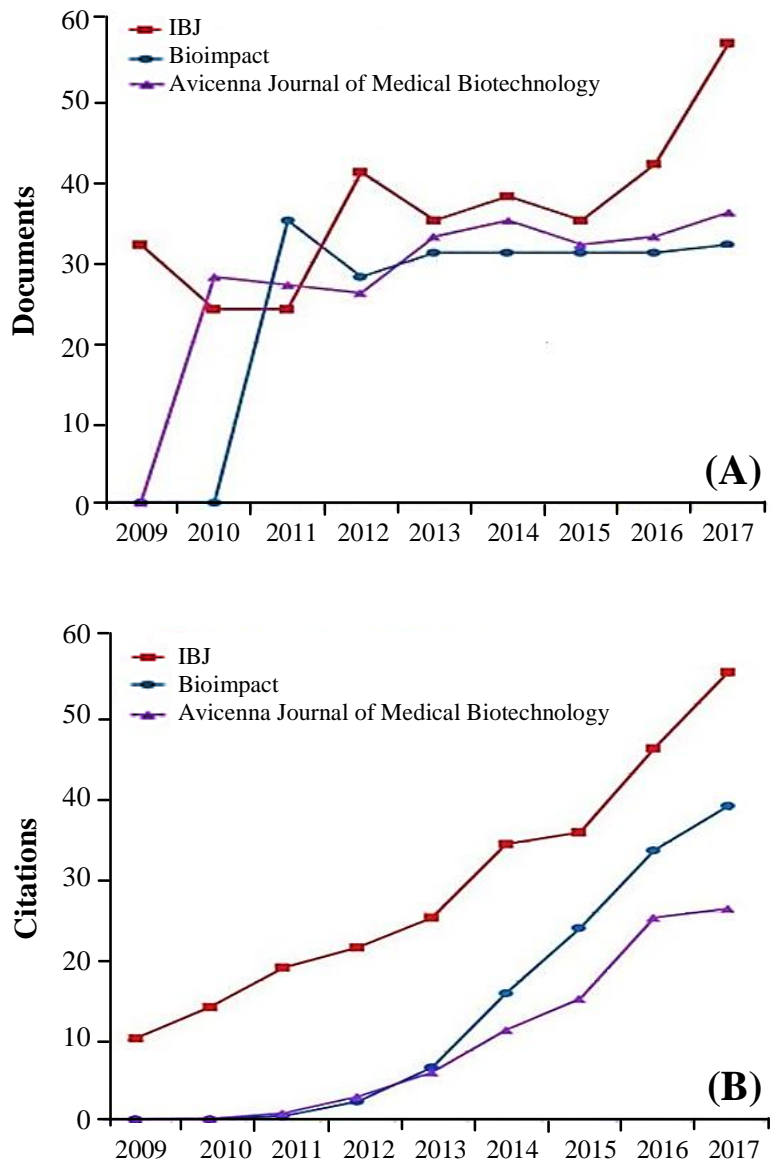

Fig. 1. (A) Document and (B) citation trends of (2009-2017) in comparison with Avicenna Journal of Medical Biotechnology and Bioimpact.

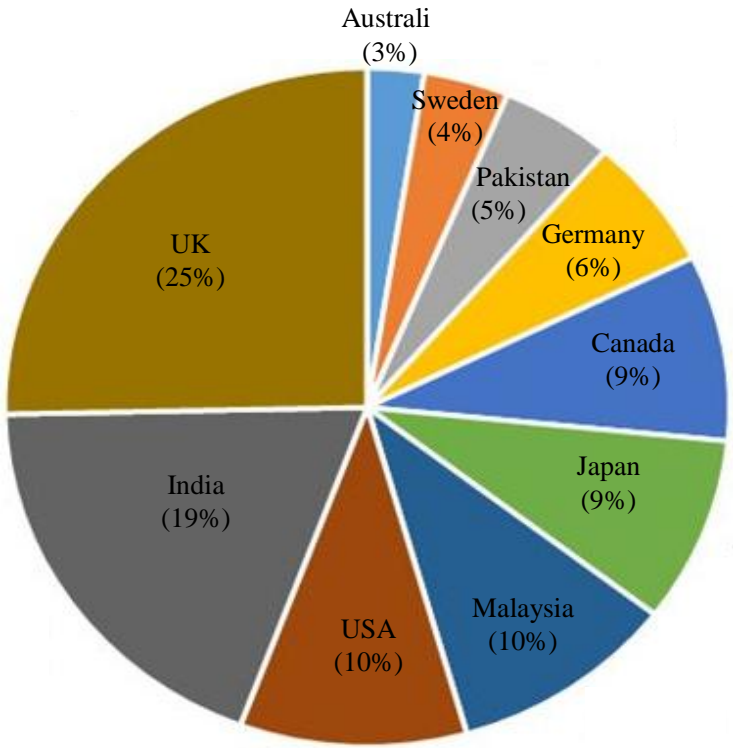

Fig. 2. The percentage of documents published in IBJ by country territories other than Iran

\section{Country territory}

Iran constituted $90 \%$ of the country territory for the 586 documents of IBJ. The remaining 10\% was distributed amongst 10 other countries, most of which belonged to UK (25\%; Fig. 2).

\section{CiteScore}

The CiteScore trend for IBJ for the period of 20112017 ranged from 0.76 to 1.92 , which peaked in 2017 (1.92; Fig. 3A). The highest trends for journals Avicenna Journal of Medical Biotechnology and Bioimpact 2017 were 1.3 and 2.46, respectively.

\section{SJR}

We assessed the SJR trend for three journals (Fig. 3B). This analysis revealed a significant rise in SJR scores for IBJ, reaching 0.574 in 2017. The highest trends for journals Avicenna Journal of Medical Biotechnology and Bioimpact 2017 were 0.441 and 0.629 , respectively.

\section{SNIP}

The analysis of SNIP scores for IBJ (Fig. 3C) revealed an increasing trend of source normalization, peaking in the years 2011 and 2107. The highest trends for the other two Journals in 2017 were 0.735 and 1.99, respectively.

\section{Q Trends}

Q trend analysis for IBJ assigned this journal mainly to Q3-Q4 in most subject areas (Table 2). However, it was able to reach Q2, in the field of biochemistry, genetics, and molecular biology, as well as medical biochemistry since 2016 . 

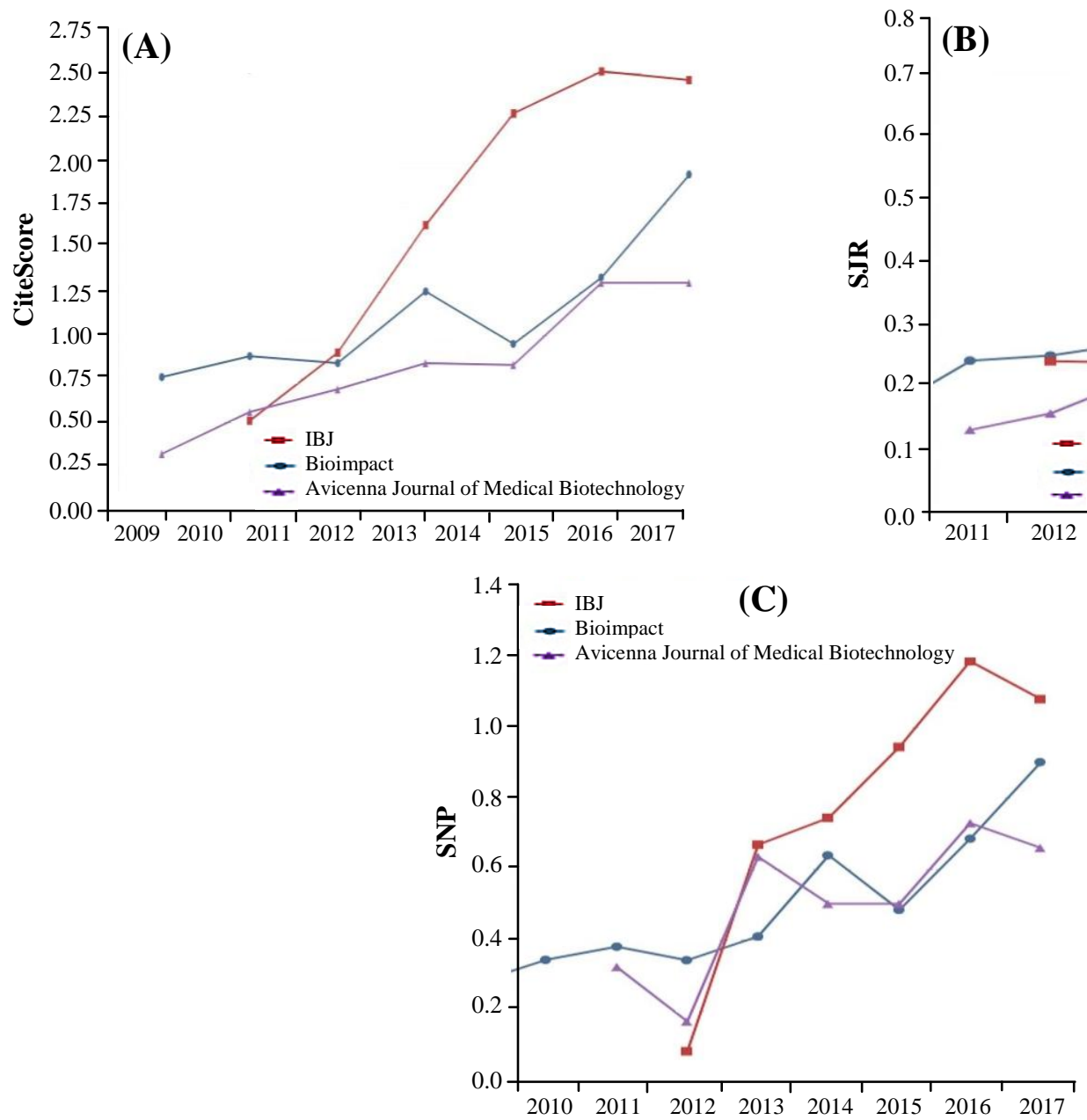

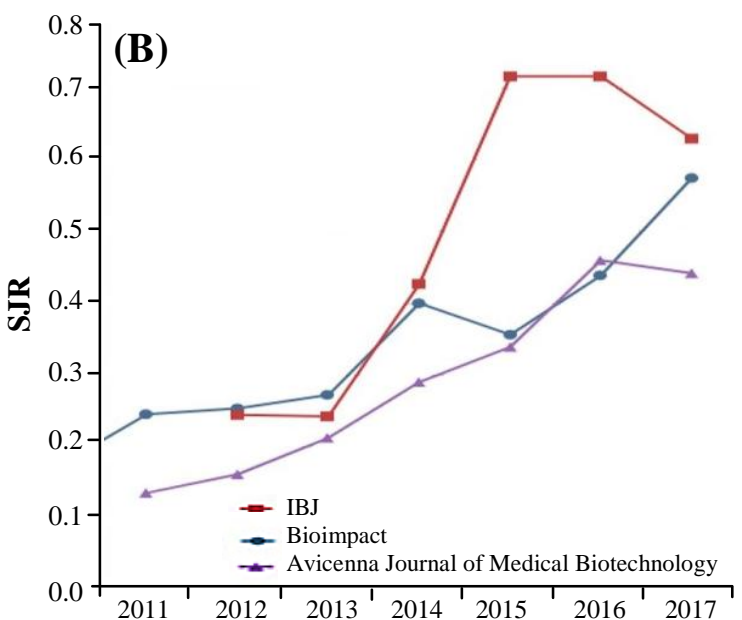

Fig. 4. (A) CiteScore, (B) SJR, (C) SNP trends for IBJ (2011-2017).

\section{RESULTS AND DISCUSSION}

The results of this paper can be very helpful for policy makers of IBJ to promote the journal activities. The scientometric analysis, presented here, has evaluated all the published documents in IBJ over a period of 18 years (2000-2017). This study reveals the journal metrics, which can be important for the authors when submitting an article to IBJ.

Scientometric studies evaluated in this paper highlight the bibliometric measures to characterize the article and journal productivity and citation analysis. The article productivity reviews the number of articles and volumes published in a journal in the past years. The journal productivity analyzes the published papers by type, subject area, and active countries in the publication of the article in a journal. The indicators of citation analyses included CiteScore, SJR, and SNIP, which help to evaluate the scientometric trend line of a journal.
Owing to the evaluated scientometric indicators and comparison with the other journals, IBJ is suggested to follow the following comments to improve its level in near future:

- Indexing the Journal in free access databases, such as EBSCOhost and DOAJ. This activity can increase the access to the published articles of IBJ, and also can increase the citation and visibility of the journal.

- Awarding an "Annual Award" to the article that receives the highest citation, without self-citation, after a one-year period of time.

- Informing the audience about the indexing databases and new indicators, such as Altmetrics, on the home page of the journal website to see this information at a glance.

- Focusing and reinforcing on the subject areas of the accepted papers to be published are important, especially in the areas of biochemistry, genetics, and molecular biology, which the journal have capacity to reach to Q1 level in Scimago database. 
Table 2. Subject area quartile (Q) of IBJ (2000-2016)

\begin{tabular}{|c|c|c|c|}
\hline \multirow[b]{2}{*}{ Year } & \multicolumn{3}{|c|}{ Subject areas } \\
\hline & $\begin{array}{l}\text { Biochemistry, } \\
\text { genetics and } \\
\text { molecular } \\
\text { biology }\end{array}$ & $\begin{array}{l}\text { Biochemistry } \\
\text { (medical) }\end{array}$ & $\begin{array}{c}\text { Clinical } \\
\text { biochemistry }\end{array}$ \\
\hline 2000 & Q4 & Q4 & Q4 \\
\hline 2001 & Q4 & Q4 & Q4 \\
\hline 2002 & Q4 & Q4 & Q4 \\
\hline 2003 & Q4 & Q4 & Q4 \\
\hline 2004 & Q4 & Q4 & Q4 \\
\hline 2005 & Q4 & Q3 & Q4 \\
\hline 2006 & Q4 & Q4 & Q4 \\
\hline 2007 & Q4 & Q3 & Q4 \\
\hline 2008 & Q4 & Q3 & Q4 \\
\hline 2009 & Q3 & Q3 & Q4 \\
\hline 2010 & Q3 & Q3 & Q4 \\
\hline 2011 & Q3 & Q3 & Q3 \\
\hline 2012 & Q3 & Q3 & Q3 \\
\hline 2013 & Q3 & Q3 & Q3 \\
\hline 2014 & Q3 & Q3 & Q3 \\
\hline 2015 & Q3 & Q3 & Q3 \\
\hline 2016 & Q2 & Q3 & Q3 \\
\hline
\end{tabular}

CONFLICT OF INTEREST. None declared.

\section{REFERENCES}

1. Gupta B, Kumar S. Citation analysis of theoretical population genetics literature. Library Herald 2001; 39(4): 208-226

2. Garfield E, Merton RK. Citation Indexing: Its Theory and Application in Science, Technology, and Humanities (Information Science S.). New York: Wiley; 1979.

3. Davarpanah M, Aslekia S. A scientometric analysis of international LIS journals: Productivity and characteristics. Scientometrics 2008; 77(1): 21-39.
4. Leydesdorff L, Opthof T. Scopus's source normalized impact per paper (SNIP) versus a journal impact factor based on fractional counting of citations. Journal of the American society for information science and technology 2010; 61(11): 2365-2369.

5. Hussain A. A Scientometric analysis of the 'Journal of King Saud University-Computer and Information Sciences' 2017; reterieved from: http://digitalcommons. unl.edu/cgi/viewcontent.cgi?article $=4364 \&$ context $=$ libp hilprac

6. Aims \& Scope. Retrieved from: https://ibj.pasteur. ac.ir

7. Espy N, Pérez-Sautu U, Ramírez de Arellano E, Negredo A, Wiley MR, Bavari S, Díaz Menendez M, Paz Sánchez-Seco M, Palacios G. Ribavirin has a demonstrable effect on Crimean-Congo hemorrhagic fever viral populations and viral load during patient treatment. The journal of infectious diseases 2018; DOI: 10.1093/infdis/jiy163.

8. Ulrichsweb global serial directory. Iranian Biomedical Journal. Retrieved from: https://ulrichsweb. serialssolutions.com/search/-244495520.

9. Mostafavi E, Bazrafshan A. Research and collaboration overview of Institut Pasteur International Network: a bibliometric approach toward research funding decisions. International journal of health policy and management 2014; 2(1): 21-28.

10. Da Silva JAT, Memon AR. CiteScore: A cite for sore eyes, or a valuable, transparent metric? Scientometrics 2017; 111(1): 553-556.

11. CiteScore ${ }^{\mathrm{TM}}$ releases 2016 annual values and updates. Retrieved from: https://www.elsevier.com/about/pressreleases/science-and-technology/citescore-releases-2016 -annual-values-and-updates.

12. Moed HF. The source-normalized impact per paper (SNIP) is a valid and sophisticated indicator of journal citation impact. Journal of American society for information science and technology 2010; DOI: 62.10.1002/asi.21424

13. Journal Metrics in Scopus: Source Normalized Impact per Paper (SNIP). Retrieved from: https://blog.scopus. com/posts/journal-metrics-in-scopus-sourcenormalized-impact-per-paper-snip SCImago Journal \& Country Rank. Iranian Biomedical Journal. Retrieved from: https://www.scimagojr.com/ journalsearch. $\mathrm{php} ? \mathrm{q}=15238 \&$ tip $=$ sid $\&$ clean $=0$. 